\title{
Sbatti e sbatta
}

\author{
Miriam Di Carlo
}

PUBBLICATO: 30 SETTEMBRE 2021

I

1 sostantivo maschile sbatti e quello femminile sbatta sono accorciamenti della parola sbattimento, deverbale disbattersi, verbo intransitivo pronominale usato in ambito colloquiale con il significato di "darsi da fare, affannarsi, faticare (anche + per o per e inf.[inito])" (Devoto-Oli 2022). L'accezione di sbattimento che ci interessa, registrata in tutti i dizionari sincronici, è quella di "il darsi da fare, anche improduttivamente, nel tentativo di ottenere un determinato risultato" e anche quella di "grande noia: che sbattimento questa serata!", marcati dal GRADIT come significati rispettivamente di ambito familiare e popolare. Per estensione sbatti e sbatta hanno anche il significato di 'voglia di fare qualcosa di estremamente noioso o fastidioso' generalmente in contesti negativi del tipo: non avere lo sbatti/la sbatta di + INFINITO (non ho lo sbatti di alzarmi dal letto). Inoltre le voci vengono utilizzate per comporre la locuzione essere/andare in sbatta/in sbatti ossia 'essere/diventare di malumore, nervoso, depresso'. Le due forme ridotte esistono da più di un decennio nel linguaggio giovanile, circoscritte prevalentemente all'area metropolitana di Milano e soltanto recentemente hanno visto un'estensione d'uso oltre questi confini d'età e geografici.

Non stupisce che il linguaggio giovanile sia proclive alla produzione di parole nuove in quanto:

una generazione produce una varietà linguistica in contrasto con la lingua comune [...]. Si presume un intenzionale distacco dalla lingua comune che garantisca la marcatezza linguistica. (Radtke i993, p. 19I)

Inoltre, riguardo all'appartenenza delle voci all'area lombarda:

i 1 parlar giovane come fenomeno nazionale non si documenta che con una tendenza di graduale sregionalizzazione di voci sempre di provenienza regionale. (Radtke I992, p. 30-I)

Dunque, come vedremo più avanti, sbatta e sbatti come accorciamenti di sbattimento nascono nel linguaggio giovanile circoscritto all'area lombarda con l'intento di creare un distacco dalla lingua comune. Il processo che si cercherà di descrivere in questo studio è proprio la "sregionalizzazione" delle voci, ossia la loro fuoriuscita dall'area di origine, per entrare poi nel linguaggio giovanile nazionale. Infine dal linguaggio giovanile, attraverso principalmente la musica si cercherà di descrivere il processo di estensione d'uso oltre i confini diastratici.

Anzitutto i due sostantivi non sono registrati in nessun dizionario sincronico dellitaliano contemporaneo, ma sbatta compare per la prima volta nel dizionario storico dei linguaggi giovanili Scrostati Gaggio! (di Renzo Ambrogio e Giovanni Casalegno, Torino, Utet libreria, 2004) in cui vengono riportati due esempi risalenti al 2002 tratti dal libro Kumpalibre dei comici milanesi Pali e Dispari. Nel 2007 entrambi i sostantivi sbatta e sbatti sono segnalati da alcuni utenti milanesi nel repertorio on line dei gergalismi giovanili Brutta storia - Manuale di lingua e mitologia urbana. Nel 2008 sbatta entra in Slangopedia, "Il primo dizionario della digital generation, teenagers anni 200o" con una segnalazione da Bergamo. Infine, viene trattato da Silverio Novelli nel 2014 in una rubrica del Magazine Lingua Italiana del portale Treccani dedicata all'approfondimento di alcune parole nuove segnalate dagli utenti del web (Silverio Novelli, I nostri lettori cacciatori di parole muove/2, treccani.it, 5/2/20I4). L'autore ne parla come di una parola ancora troppo relegata all'ambito giovanile della città 
di Milano e quindi non considerabile un neologismo stricto sensu. Ad oggi invece possiamo dire che, a distanza di meno di sette anni, sbatti e sbatta si sono diffusi, seppur non omogeneamente, su tutto il territorio della nostra penisola, prevalentemente in ambiti informali e giocosi, almeno fino alla generazione dei quarantenni. Le occorrenze sul web sono comunque ancora molto contenute: per la stringa "uno sbatti" Google restituisce 5.080 risultati mentre per "una sbatta" 3.860; per "in sbatti" 5.970 mentre per "in sbatta" I.400 (ricerche del I6/09/202I). Risulta improduttivo fare la ricerca per le parole senza articoli o con gli articoli determinativi perché si ottengono tutte le occorrenze in cui si ha omonimia con le forme coniugate del verbo sbattere, accompagnate eventualmente dai clitici lo (= lui) e la $(=l e i)$.

Per quanto riguarda il significato, come si accennava in apertura, sbatti e sbatta provengono dal verbo sbattersi con valore figurato e familiare di 'darsi da fare, faticare, affannarsi' attraverso il deverbale sbattimento 'lavorio affannoso, frenetico'. Per estensione sbattimento ha assunto, come si è detto all'inizio, anche l'accezione di 'situazione noiosa, barba, pizza' (Devoto-Oli 2022) soltanto in tempi relativamente recenti, in quanto le due accezioni non sono registrate nel GDLI (il volume che contiene la parola sbattimento è stato pubblicato nel I994; sbattimento è assente nei due Supplementi del 2004 e 2009). Il GDLI, che è un dizionario che si basa sulle fonti letterarie, registra insieme due ulteriori significati di sbattimento (entrambi assenti nei dizionari più aggiornati come il Devoto-Oli 2022 e lo Zingarelli 2022), il primo specifico del gergo dei tossicodipendenti e il secondo, considerato derivato dal primo, di carattere più generale:

gerg.[ale] Ricerca affannata e frenetica di droghe o di altre sostanze stupefacenti, soprattutto in momenti di crisi di astinenza. - Ricerca disordinata di uno stile di vita non convenzionale, di moduli culturali in contrasto con le consuetudini e le tradizioni.

La citazione riportata dal GDLI con riferimento al secondo significato, è di Alberto Arbasino, autore lombardo:

Dopo anni di ricerche e di sbattimenti, troveranno 'alternativo' tutto ciò che era ovvio per nonna Carolin, a partire dal suo nome: le sale da tè con crostate di frutta e il vino proprio, pigiato nel podere, gli abiti lunghi della bisnonna e i gilets neri del bisnonno, il cherleston della mamma e il rock 'n' roll della zia. (Alberto Arbasino, Un paese senza, Milano, Garzanti, 1980, p. I30)

Dalla documentazione disponibile non è possibile stabilire se quest'uso della parola sbattimento, pur essendo significativo perché gergale e diffuso nell'ambito giovanile, sia in correlazione con gli accorciamenti sbatti/sbatta. Appare invece evidente che i due accorciamenti siano collegati a locuzioni come sbattimento di palle o sbattimento di coglioni, di registro colloquiale basso, già diffuse in ambito per lo più giovanile, per indicare una sensazione di pesantezza, noia o fatica:

Qualche volta ci sono andata, a trovarla. Lei mi voleva portare ai concerti di musica classica, sai che sbattimento di palle. (Melania Gaia Mazzucco, La vita assassina, Torino, Rai.eri, 1997, p. 2I)

Dal punto di vista morfologico, sbatti va considerato un vero e proprio accorciamento di sbattimento (come frigo difrigorifero e tele d itelevisione) mentre sbatta potrebbe derivare da sbatti, con un metaplasmo di genere e un cambio di desinenza, su spinta dei sinonimi noia, pizza, barba. In quanto di formazione recente, sbatta non si può considerare né una conversione da sbattere (come chiama dà chiamare), né una riduzione di un nome femminile in -zione (come qualifica da qualificazione, visto che non è documentato "sbattizione; c'e sbattitura, ma in genere questi deverbali non sono coinvolti nel processo), ma semmai una retroformazione. Il fenomeno dell'accorciamento (assieme a quello della 
retroformazione) è caratteristico del linguaggio giovanile: basti pensare alle forme allocutive come prof per professore, fra per fratello, raga per ragazzi/e e anche matusa per matusalemme, tranqui per tranquillo, i più datati spino e siga per spinello e sigaretta (D'Achille 2006, p. I99).

Le prime attestazioni trovate sul web di sbatti e sbatta sono pressappoco coeve visto che la prima occorrenza di sbatti risale al 2003 mentre quella di sbatta al 2004:

piuttosto sapete mica dove trovare dei manga scan online? Almeno mi evito lo sbatti di prendere il manga incompletyo [sic] x la parte finale :) (commento di kyosuke8I sul forum hwupgrade del $27 / 8 / 2003)$

per quanto riguarda le cartine, esiste un tipo di smoking realizzato interamente in canapa, con colla naturale. Non credo esista la "versione" corta da sigaretta, ma alla fine basta tagliarle no? Dite che è una sbatta? Vabè :p (commento di nighthe del 4/5/2004 su forumhwupgrade.it)

Ma la precedenza di sbatti sembra confermata dal fatto che ha una quantità di attestazioni molto maggiore: già nel 2003 compare in alcuni testi rap di autori milanesi come ad esempio Se voi ci capireste, scritto da Bassi Maestro e Meddaman:

Fatti quattro passi con Busdeez, tranqui, ti rilassi

Che a conti fatti, più sbatti, siamo quattro gatti

(testo di Se voi ci capireste di Bassi Maestro e Meddaman di Marzo 2003 sul sito genius.com)

Negli anni successivi, sbatti continua ad essere usato dai rapper milanesi o appartenenti all'area settentrionale d'Italia, come ad esempio in Questo fuoco, i cui autori, Zampa e Mistaman, sono entrambi veneti, uno di Verona e l'altro di Treviso:

A volte sì, yo, sapessi i dubbi che ho gli sbatti che ho

I viaggi nella testa che mi assalgono brò

(testo di Questo Fuoco di Zampa e Mistaman del 25/3/2004 sul sito genius.com)

Nel 2006 esce il brano di Zampa in cui sbatti (al plurale) compare addirittura nel titolo, Mille sbatti per l'appunto:

Vivo in un mare di sbatti [...]

Gli altri vanno avanti schiavi di mille sbatti [...]

Se, vivi come me,

tu ne senti il peso, resti teso tra sbatti

(testo Mille sbatti di Zampa del 2006 sul sito angolotesti.it)

Solo per citare qualche brano rap in cui compare sbatti e dare unidea dell'incidenza della parola nei testi appartenenti a questo genere musicale, si segnala che nel 2007 esce il brano Dolce Paranoia del Club Dogo, gruppo milanese, seguito poi, nel 2009 da altri due brani Richiamami domani e Boing. Del 2or è il disco di Fedez (anche lui milanese) Il mio primo disco da venduto, in cui compare il brano Blues, composto insieme al milanese Guè Pequeno e al siciliano Marracash e il quale uscirà come singolo nel 20I2: nella parte cantata da Fedez abbiamo la frase "Lo sbatti di mettere insieme una somma". Sempre a questi anni appartiene il brano Un abbraccio e ti amo di GionnyScandal, il quale, a differenza di tutti gli altri rapper finora citati, è originario di Pisticci, cittadina della Basilicata (provincia di Matera). Notiamo una certa continuità nell'uso di sbatti all'interno dei brani rap, con un netto incremento a partire dal 2015, anno in cui compare in After di Giaime (anche lui di Milano), in molti brani scritti dal 
milanese Sfera Ebbasta (alcuni assieme a Charlie Charles, anche lui di area milanese) come Te lo do e in Tutti scemi. Fuori dal genere rap, sbatti viene usato come titolo di una canzone (preceduto dall'hastag: \#Sbatti) del gruppo milanese Il Pagante, il quale raggiunge la sua popolarità tra il 2012 e il 20r6. Nel 2018 è di nuovo (al plurale) nel titolo di una canzone del romano Gazelle, scritta assieme al compositore romano Federico Nardelli: "e ci arriveremo tardi a tutti i nostri sbatti".

Sul web sbatti registra un incremento d'uso che riflette verosimilmente la crescente popolarità che i rapper hanno raggiunto nell'ultimo decennio (ricerche sulle pagine in italiano di Google del 20/9/2O2I):

\begin{tabular}{|l|l|l|l|}
\hline anno & "uno sbatti" & anno & "uno sbatti" \\
\hline 2003 & 1 & 2013 & 49 \\
\hline 2004 & 1 & 2014 & 38 \\
\hline 2005 & 2 & 2015 & 108 \\
\hline 2006 & 5 & 2016 & 72 \\
\hline 2007 & 6 & 2017 & 57 \\
\hline 2008 & 16 & 2018 & 89 \\
\hline 2009 & 9 & 2019 & 115 \\
\hline 2010 & 10 & 2020 & 88 \\
\hline 2011 & 20 & 2021 & 102 \\
\hline 2012 & 22 & & \\
\hline
\end{tabular}

Nonostante i dati siano puramente indicativi di una tendenza e la crescita presenti dei punti di discontinuità, è comunque evidente che, a partire dal 2015 circa, il sostantivo sbatti comincia ad avere un uso più consistente (non abbiamo preso in considerazione "lo sbatti" e "gli sbatti" per i motivi già espressi in precedenza).

Controllando le varie occorrenze di sbatti, vediamo che viene impiegato principalmente sui forum o comunque all'interno di commenti sui blog ossia in una modalità digitata di conversazione informale. Si tratta inoltre ancora di forum frequentati prevalentemente da giovani: si va da conversazioni sui videogiochi, a quelle sui manga, sui motori, sui trucchi:

Eccomi!!! :D

Semplicemente non mi era ancora stato abilitato l'account...chiedo scusa per lo sbatti a oveja e ringrazio anticipatamente pap per il logo! (commento di AlphMaster sul forum htita.it del 23/4/2005)

Grandissimo!!!

E [sic] veramente gentilissimo anche per lo sbatti di uploadare le immaginei [sic]!!!

(commento di Haitiz sul forum treddi.com del 22/II/2006)

Bravo finalmente recensisci qualcosa di semi-intelligente! Va be' recensione troppo lunga è sbatti da leggere...meglio quello di Malgioglio se fossi DrAdder ti darei 0.5 periodico...comunque album caruccio! (commento di CarlMarx sul forum debaser.it del 27/4/2008)

Le occorrenze di sbatti rimangono circoscritte alle conversazioni sui forum, per lo più usato da persone di area milanese o settentrionale almeno fino al 2016-2017 quando le attestazioni coinvolgono alcuni veri e propri siti: 
Perché il mattino avrà pure l'oro in bocca, ma lo SBATTI E' OVUNQUE! Insomma uno SBATTI, DA DIETRO E DA DAVANTI. (Guido Dandrea, Figa se oggi è in salita! Tutti gli sbatti di prima mattina, imbruttito.com, 23/10/2017)

Un servizio semplice, senza pretese, che tramite uninterfaccia sbarazzina (paletta cromatica palesemente copiata da youtube) ci fornisce tutto ciò di cui abbiamo bisogno per creare una persona nuova (senza lo sbatti di dover trovare una persona del sesso opposto al nostro e copularci e bla bla bla) (Mattr7, 5 consigli utili che (forse) non conoscevi, leganerd.com, 2017)

Celebrate ma soprattutto rispettate lo sbatti del vostro partner (Papaperscelta, I 10 Papocomandamenti, papaperscelta.it, 28/3/2018)

Il pagante di solito viene da una famiglia conservatrice, ma se ne frega di tutte le cose che comportano impegno e fatica (lo sbatti). (Edoardo Cremona, Il Pagante: "Prendete pure in giro i paninari 2.o, ma saremo la classe dirigente di domani", linkiesta.it, 26/r/2019)

Non possiamo dire che sbatti abbia un'incidenza vera e propria sui giornali, e sicuramente non appartiene al repertorio lessicale dei giornalisti. Infatti compare in un'intervista a un rapper milanese:

Ero l'ultima ruota del carro. Ho vissuto male questa cosa della discografia, perché è stato uno sbatti (Antonio Dikele Distefano, Il mio incontro con Ghali, "rapper di quartiere" tra Baggio e la Tunisia, lacittanuova.milano.corriere.it, 29/6/2015)

Anche l'articolo della "Repubblica", in cui sbatti viene enfatizzato graficamente con l'uso delle virgolette, appartiene all'edizione del capoluogo lombardo (come quello del "Corriere" riportato precedentemente) mentre uno della "Stampa" riporta la citazione di un post:

è stato da quel momento che abbiamo iniziato ad apprezzare l'importanza delle piccole cose, anche di ciò che consideravamo un fastidio, uno "sbatti": le giornate di pioggia con la metro affollatissima e i chili di segatura sparsi per le scale del liceo, segatura che ti si appiccica ovunque e te la ritrovi persino nei capelli, le macchinette in cui è sempre bloccata l'unica merendina che volevi tu e le casse della lavagna elettronica che ogni tanto [...] emettono un fischio acutissimo, che ti trapana per bene i timpani. (Carola Pardi, Come realizzare la foto di classe quando si è reclusi in casa? La soluzione degli studenti di Milano, milano.repubblica.it, 28/4/2020)

"Far passare l'homeworking come una grande opportunità, ma senza fare i conti con lo sbatti che implica, è una presa in giro" - si legge ancora nel post -. (Luisa Mosello, Picchia la madre dei suoi figli mentre loro sono in Dad: denunciato, lastampa.it, 2I/3/202I)

Comunque, il fatto che il termine venga usato in testate giornalistiche di diffusione nazionale significa che si pensa che gli italiani possano comprenderlo, oppure che si vuole dare una coloritura attuale e giovanile al pezzo. Attraverso una ricerca su Google Libri notiamo che già nel 2or2 sbatti viene usato nei titoli, per lo più in riferimento al lessico giovanile o comunque in maniera giocosa (Mariacristina Ferrario, Che sbatti oggi balza. L'adolescenza: un mondo di immense possibilità, Milano, Megalini, 20I2; Francisco Paolo Quilici, Le ciccionate. 140 ricette esagerate, low cost e senza "sbatti", Milano, Rizzoli, 2013). Nel 2017 esce, sempre per Rizzoli, il libro del già ricordato rapper lucano GionnyScandal il cui lessico, inevitabilmente impregnato di gergo giovanile, propone ripetutamente sbatti:

Perché arriva sempre, arriverà anche oggi, il momento in cui mi prende l'ansia, vado in sbatti e non riesco a pensare a nient'altro. [...] Solitamente in questi casi preferisco non rispondere, anche su Facebook dopo un po' ho deciso di chiudere la bacheca per evitare troppi sbatti inutili. (GionnyScandal, La via di casa mia, Milano, Rizzoli, 20I7) 
Due anni dopo, esce il libro dell'insegnante Davide Tamaglini il quale, descrivendo la sua esperienza di docenza a Milano, riporta sbatti all'interno di alcune battute pronunciate da ragazzi:

"Tutto 'sto sbatti per scrivere un testo di presentazione all'esame!?!"

"Non gliene frega niente a nessuno di come siamo diventati 'sto schifo!"

(Davide Tamaglini, Continueró a sognarvi grandi. Storia di una rivoluzione tra i banchi di scuola, Milano,

Longanesi, 2019)

Infine, nel 202I, compare nel titolo della traduzione di Valeria Prezioso al libro della nutrizionista americana Kate Hamilton, Chetogenica Zero Sbatti, uscito nel 202г.

In definitiva, le occorrenze sui quotidiani, quelle sui libri e l'uso nel titolo della canzone del romano Gazelle e all'interno del libro del rapper lucano GionnyScandal ci dicono che sbatti tende orami a superare il confine che separa il gergo giovanile dell'area metropolitana di Milano (o comunque lombarda) dal registro più colloquiale e familiare del lessico italiano.

Leggermente differente è la storia disbatta che, come si è detto, sembrerebbe essere nato successivamente, per derivazione da sbatti, nello stesso ambito, per poi fuoriuscire dal gergo giovanile milanese più velocemente e più nettamente. Assieme a sbatti compare nel 2007 nel repertorio dei gergalismi giovanili compilata direttamente dagli utenti, Brutta Storia - Manuale di mitologia urbana. $\mathrm{Su}$ Twitter comincia a comparire nel 2008, con 5 tweet nei quali sempre presenta l'estensione di significato 'noia, pizza' e poi 'voglia di fare qualcosa di noioso' che spiegherebbe, come si è detto, il metaplasmo di genere al femminile (non tutti i tweet hanno il link perché non sono più visibili singolarmente):

Vi segnala Mixwit. Come Muxtape, ma senza la sbatta di caricare le canzoni. Roba da perderci le notti. O no? (tweet di @kekkoz del I2/6/2008)

LOL!! Con scp non è possibile? So che ci sono dei server anche per Win... Mispiace per la sbatta (tweet di @Blazar del 23/9/2008)

se non ci fosse la sbatta di andare fino a Verno lo farei volentieri. Per alimentare il mio gusto per l'improbabile (tweet di @seze del 28/10/2008)

che poi con tutta la sbatta che c'è le fanpage potevano pure aspettare eh..... (tweet di @Al_bo del $8 / \mathrm{IO} / 2008)$

ho trovato la sbatta per cercare sfondi $x$ il suo iPhone e mettergli una suoneria decente (tweet di @matteomaggioni del 2/II/2008)

Per la maggior parte, i testi in cui viene usato sbatta sono di carattere colloquiale, caratterizzati per lo più da un registro basso o informale come i commenti sui forum o sui blog:

Si può giocare acneh [sic] via Internet così non dobbiamo più fare la sbatta di trovrci tutti a casa mia! Caste sarà contento (se riuscisse a collegarsi a Internet...) (commento di Cody hell del 9/I/2006 sul forum freeforumzone.it il forum della Compagnia del Monte)

cioè: una multinazionale con più di Io.ooo negozi nel mondo ha un sistema così chirurgico che si fa la sbatta di mandare lettere in giro per racimolare SEI sterline che gli avrei rubato in sconti (commento di Ganja del 3/r7/2008 sul forum netgamers.it) 
Mi spiace per la sbatta che vi ho fatto fare (ma erano pochi interventi e c'e il dio ctrl+F che aiuta) ma lo reputo un punto importante, ancora di più di una discussione nella quale fra l'altro s'è parlato di digitale (commento di Elvezio del 5/7/2010 sul forum loredanalipperini.blog.kataweb.it)

Al pari di sbatti, nel zoog e poi nel 20II, sbatta viene impiegato in due testi rap di J-Ax, anche lui come altri citati in precedenza di origine milanese ma che, diversamente da loro, al momento dell'uscita dei pezzi in questione già aveva una notorietà tale da far supporre che le sue canzoni fossero ascoltate su tutto il territorio nazionale:

Mantiene la calma davanti all'ispezione
Io invece vado in sbatta, ho la palpitazione
(testo de Il Commercialista uscito nell'album Deca Dance di J-Ax del 2009 sul sito testicanzoni.mtv.it)

Musica da sbatta e chissenefrega se la radio la passa o no!

Musica da rabbia / per chi c'ha la sbatta / per chi c'ha la sbatta

(testo di Musica da rabbia di J-Ax del 20II sul sito genius.it)

Dal punto di vista semantico nel primo testo andare in sbatta equivale a 'essere nervoso, agitato' mentre nel secondo avere la sbatta 'essere arrabbiato'; dunque sbatta significherebbe tanto 'agitazione' (anche in uno stato di soggezione) quanto 'rabbia' (in uno stato più aggressivo).

A partire dal 2orr la parola comincia timidamente a uscire dai testi di parlato digitato e a comparire in altri dal carattere più complesso, come articoli o post ben strutturati:

Accertata la necessità della maschera, adesso avete due possibilità: comprarla, oppure farvela da soli. Per quelli che hanno voglia di farsi la sbatta (e che saranno premiati per l'impegno dimostrato diventando meno poveri e più belli) c'è una curiosa maschera che tutti noi possiamo fare a casa, soprattutto se abbiamo un gatto. (Elisa Colonna, La Trousse: maschera di bellezza, vice.com, I2/I2/20II)

La difficoltà peggiore per chi ha una relazione a distanza non sono i primi chilometri, la scarsa qualità delle chiamate Skype, la solitudine prima di andare a letto o il fai-da-te obbligatorio - ma le persone che pongono noiosissime domande su come si fa ad avere un rapporto a distanza. Quindi ecco una sintetica ma comunque straziante carrellata di questioni troppo spesso poste a chi già deve sorbirsi la sbatta di avere obblighi di un fidanzato e le tristezze di un single. (Agostino Bertolin, 15 cose che chi ha una relazione a distanza è stufi di sentirsi chiedere, oltreuomo.com, 2015)

Come detto in apertura, nel 2014 il Magazine del portale Treccani dedicato alle parole nuove segnalate dagli utenti decide di trattare sbatta e non sbatti, pur sottolineandone la circolazione limitata all'area lombarda e all'ambito giovanile. A differenza di sbatti, sbatta compare sui giornali nazionali e non nelle edizioni lombarde seppure, nella maggior parte dei casi, o con un uso gergale o con un riferimento alle varietà regionali settentrionali:

In un'altra inchiesta, coordinata dal sostituto procuratore [...], emerge come queste siano vacche da mungere. In una conversazione whatsapp di un altro gruppo, attivo tra Bovisio Masciago e altri comuni brianzoli, i carabinieri hanno ricostruito un altro meccanismo di approvvigionamento degli anabolizzanti, ovvero quello di mandare dei "cavallini" a setacciare le farmacie presentando ricette mediche contraffatte. «Hai voglia di fare una sbatta per 25 euro? », si legge in uno dei messaggi. (Luca De Vito, Cosi agisce il racket dei muscoli gonfiati, repubblica.it, 5/3/2020)

Alle 22 il Blah Blah in via Po 2r Max McMorte e i Satanisti progressisti propongono il peggio dello «Shock-Rock» sulla piazza. Il «Tour Nefasta Taurinorum» dissacra il centro di Torino e annuncia al mondo che «il disco è quasi finito e non lo ristampiamo perché è una sbatta». ([s.f.], S.t., repubblica.it, 
I $8 / 4 / 2014)$

Allora, io ho affittato mia sorella alla Pantene firmando tutte le clausole per sicurezza ho accettato le condizioni di massima offerte dal piano di estrazione bulbare, l'implantologia Cordyceps fruttifica senza sbatta eccessiva procederà lei al login [...] vuoi tu prendere in TSO baraccopoli questo rantolo da Villaggio Brollo fino a Sovico nel bene e nei pacchi alimentari nei TFR e nei frontali ad autoscatto? (Davie Albanese, Adagi, repubblica.it, I6/I/202I)

La differenza sostanziale con sbatti sta nel fatto che, negli ultimi due anni, sbatta registra un uso crescente, sia all'interno di interviste e post strutturati, sia nei libri, senza alcuna marcatezza milanese:

Dalle magliette ufficiali ai volti dei giocatori, passando per i nomi più antichi degli stadi fino al magico Bologna del $1935 / 36$. Una sbatta tremenda che merita di essere ripagata con la giusta popolarità. (L'uomo che ha riprodotto su PES 6 la Serie A dal 1929 ad oggi, calciatoribrutti.com, I4/6/2019)

Risponde il conduttore [Alessandro Cattelan], "ma non ho voglia di farmi la sbatta di cambiarle" (Morgan K. Barraco, Alessandro Cattelan/ "Chi è realmente la persona piu carina della to italiana?", il sussidiario.net, $17 / 9 / 2020$ )

Che poi montare il ring è una sbatta allucinante: scaricare il furgone, le travi, le tavole, le corde, il pezzo che manca (Beniamino Malacarne, Quando ti manca il wrestling, anche una gomitata in bocca fa piacere, oblaoaps.it, 28/6/202I)

Meglio togliersi la sbatta il primo giorno e poi sparire che ricevere continuamente il tipico messaggio che chiede "Quando passi" e il punto di domanda. (Paolo Bontempo, Gianluca Dario Rota, Giugno, Sperling \& Kupfer, 2020)

La prima lo aveva lasciato due anni di distanza dalla nascita del secondo figlio - quando la sbatta del non dormire un cazzo era finita [...] Harry aveva implorato - a suo modo - Alex di fare un ultimo turno al pub, una sera consacrata di diritto a Janice, perché non c'aveva la sbatta di farsela [...] Al Borgo non avevano la sbatta di andare a rompere i coglioni fuori dal loro piccolo territorio. (Davide Catena, Gine Cocaina, Youcanprint, 202I)

Confrontiamo infine sbatta e sbatti nelle occorrenze su Google (pagine in italiano, ricerca del 28/9/2O2I):

\begin{tabular}{|c|c|c|c|}
\hline \multicolumn{2}{|c|}{ sbatti } & \multicolumn{2}{c|}{ sbatta } \\
\hline "uno sbatti" & "in sbatti" & "una sbatta" & "in sbatta" \\
\hline 11.500 & 5.920 & 3.240 & 1.620 \\
\hline
\end{tabular}

Nel 202I (fino al 28/9) sbatta risulta avere ancora meno occorrenze rispetto a sbatti ("una sbatta" 39 r., "uno sbatti" II7 r.) ma, se si confrontano le varie attestazioni, si nota che sbatta viene usato con più frequenza fuori dall'ambito lombardo rispetto a sbatti, che invece continua a comparire in siti dedicati alla realtà milanese (come imbruttito.com e yesmilano.it). Forse la terminazione in $-i$ al singolare maschile e quindi l'invariabilità di sbatti costituisce un freno alla sua espansione, laddove sbatta si inserisce meglio nella tradizionale morfologia nominale italiana.

In definitiva, il sostantivo sbatti rimane una parola nuova ancora troppo ancorata al contesto milanese e giovanile e dunque non trattabile come vero e proprio neologismo. Invece, vista l'incidenza sui quotidiani nelle edizioni nazionali e sui libri editi di recente, possiamo considerare sbatta una nuova 
parola che sta emergendo lentamente nel repertorio lessicale di una consistente parte di italiani e dunque più vicina alla categoria di neologismo. Inoltre la complessità semantica dei termini, i quali continuano ad arricchirsi di sfaccettature nuove di significato e a formare locuzioni, denota la vitalità d'uso di entrambi. Resta in ogni caso un fenomeno interessante, da monitorare per coglierne le future evoluzioni sia semantiche sia di distribuzione areale.

Nota bibliografica:

- Michele A. Cortelazzo, La componente dialettale nella lingua delle giovani e dei giovani, in Gianna Marcato (a cura di), Donna \& Linguaggio, Padova, Cleup, 1995, pp. 58I-586.

- Lorenzo Coveri, Una lingua per crescere, Firenze, Cesati, 2014.

- Paolo D’Achille, L'italiano contemporaneo, Bologna, Il Mulino, 20o6, pp. 198-20o.

- Carla Marcato, I gerghi italiani, Bologna, Il Mulino, 2orz.

- Edgar Radtke, Varietá giovanili, in Alberto A. Sobrero, Introduzione allitaliano contemporaneo (La variazione e gli usi), Roma-Bari, Laterza, I993.

- Edgar Radtke, La dimensione internazionale del linguaggio giovanile, in Emanuele, Banfi, Alberto A. Sobrero (a cura di), Il linguaggio giovanile degli anni Novanta, Roma-Bari, Laterza, I992, pp. 5-44.

\section{Cita come:}

Miriam Di Carlo, Sbatti e sbatta, "Italiano digitale", XVIII, 2021/3 (luglio-settembre)

DOI: $10.35948 / 2532-9006 / 2021.11613$ 